\title{
Compton Scattering and Nucleon Polarisabilities: Theory Progress
}

\section{Harald W. Grießhammer*}

Institute for Nuclear Studies, Department of Physics, George Washington University,

Washington DC 20052, USA; E-mail: hgriedgwu . edu

\section{Judith A. McGovern}

School of Physics and Astronomy, The University of Manchester, Manchester M13 9PL, UK

\section{Daniel R. Phillips}

Department of Physics and Astronomy, Institute of Nuclear and Particle Physics,

Ohio University, Athens $\mathrm{OH} 45701$, USA

We consider the response of nucleons and light nuclei to electromagnetic fields to test the ability of Chiral Effective Field Theory to describe such systems in a consistent and systematicallyimprovable manner.

The 9th International workshop on Chiral Dynamics 17-21 September 2018

Durham, NC, USA

\footnotetext{
* Speaker.
} 


\section{Introduction}

At sufficiently low energy, gauge and Lorentz invariance require that Compton scattering from a composite target reduces to Thomson scattering, depending only on the charge and mass. At somewhat higher energies, deviations become apparent, and the form of these deviations is a sensitive test of the important low-energy degrees of freedom determining the target's structure. The aims of Compton scattering experiments from light nuclei at photon energies around 50-150 MeV are therefore two-fold: an exploration both of nucleon and of nuclear structure. For a proton target, the principal such effects at low energy (after well-undestood relativistic corrections, $\pi^{0}$ pole contributions and the anomalous magnetic moment) are the nucleon's electric and magnetic dipole polarisabilities, $\alpha_{E 1}$ and $\beta_{M 1}$. These reveal the extent to which charge and current distributions in the target shift under the influence of external electromagnetic fields and parametrise the strength of the induced radiation dipoles. Then, in the amplitudes that are sensitive to the target's spin, four "spin polarisabilities" $\gamma_{i}$ govern the departure from point-like scattering and parametrise the response of the spin degrees of freedom. For light nuclei $\left(\mathrm{d},{ }^{3} \mathrm{He},{ }^{4} \mathrm{He}\right)$ the deviations from Thomson scattering set in much earlier, at energies around the break-up energy, and by $50 \mathrm{MeV}$ the response involves an interplay of both nucleonic and nuclear excitations. A satisfactory description of such experiments both validates the underlying theory, and allows for an extraction of the otherwise-inaccessible neutron polarisabilities [1].

For reference we reproduce the low-energy non-relativistic effective Hamiltonian that indicates how polarisabilities affect the response of the nucleon to external electric and magnetic fields [2]:

$$
\begin{aligned}
H_{\mathrm{eff}}=-\frac{1}{2} 4 \pi( & \alpha_{E 1} \vec{E}^{2}+\beta_{M 1} \vec{B}^{2} \\
& \left.+\gamma_{E 1 E 1} \vec{\sigma} \cdot \vec{E} \times \overrightarrow{\dot{E}}+\gamma_{M 1 M 1} \vec{\sigma} \cdot \vec{B} \times \vec{B}-2 \gamma_{M 1 E 2} E_{i j} \sigma_{i} B_{j}+2 \gamma_{E 1 M 2} B_{i j} \sigma_{i} E_{j}\right),
\end{aligned}
$$

where dots mean a time derivative and $X_{i j}=\frac{1}{2}\left(\nabla_{i} X_{j}+\nabla_{j} X_{i}\right)$. The scalar polarisabilities will be given throughout in units of $10^{-4} \mathrm{fm}^{3}$, and the spin polarisabilities in units of $10^{-4} \mathrm{fm}^{4}$.

At this conference, plenary and parallel talks by D. Hornidge, E. Downie, A. Alexandru, Ph. Martel, M. Sikora, L. Myers, R. Miskimen, C. Howell, M. Hoferichter, A. Walker-Loud, R. Young and V. Lensky [3] discussed the large-scale international effort, gains and goals of a new generation of high-precision facilities to understand low-energy Nuclear Physics by extracting nucleon polarisabilities from Compton scattering experiments. Some highlight the importance of electromagnetic polarisabilities in many contexts; others showed that determining them by experiments takes years of planning, execution and analysis - and commensurate theory support.

In recent years there has indeed been an upsurge in interest in the polarisabilities of the nucleon, both scalar and spin, with a number of new experiments planned, running or completed at MAX-Lab, MAMI and HI $\gamma \mathrm{S}[4,5,6,7,8]$. In addition the magnetic polarisability $\beta_{M 1}^{(\mathrm{p})}$ has been shown to be a crucial input in the determination of the two-photon-exchange contribution to the Lamb shift in muonic hydrogen and the isovector $\beta_{M 1}^{(\mathrm{p})}-\beta_{M 1}^{(\mathrm{n})}$ has been connected to the nucleon electromagnetic mass difference (see references in [9]). The calculation of nucleon polarisabilities is also an aim of lattice QCD, and several groups now have published results, albeit almost all at large pion masses (see references in [10]). In this contribution we report on the results of highprecision EFT fits of scalar polarisabilities to current data, and on prospects for the determination 
of spin polarisabilities. For more details and in particular for more complete references to earlier work the reader is referred to our review [1], and to subsequent papers $[6,9,10,11]$, and another contribution on ${ }^{3} \mathrm{He}$ in these proceedings [12].

\section{Chiral Effective Field Theory}

The framework we use in our calculations is based on the extension of chiral perturbation theory to the one- and few-nucleon sector [13]. This Chiral Effective Field Theory involves hadronic degrees of freedom rather than elementary quarks and gluons. It exploits the full symmetry of the QCD Lagrangian which arises from the fact that the up and down quarks are not only close in mass (hence isospin symmetry) but also very light compared to typical hadronic masses. Contrary to what one may read, the mass of ordinary matter does not arise primarily from coupling to the Higgs field. In fact if that were turned off, the proton and neutron would remain massive and would not change dramatically. In that limit the symmetry of isospin would be doubled, with one copy for each of the unmixed massless left- and right-handed quarks: the assumption of chiral perturbation theory is that we can treat the real world by expanding perturbatively about that limit. The symmetry is realised in the hidden mode, so the hadron spectrum does not contain degenerate parity doublets; instead pions, which are much lighter than other hadrons, can be interpreted as Goldstone bosons, massless in the chiral limit. The symmetry also requires their interactions with one another and with other hadrons to vanish in the low-momentum (soft) limit. Hence, somewhat surprisingly, a theory of the strong interaction turns into a theory of weakly interacting hadrons, provided we confine ourselves to energies below some breakdown scale, and in practice not too much greater than the pion mass. Being a field theory, the incorporation of coupling to photons (and indeed $\mathrm{W}$ and $\mathrm{Z}$ bosons) is straightforward, and gauge-invariance is built in. The theory is renormalisable order-by-order in the following sense. The Lagrangian consists of infinitely many terms but with a finite number at each order in a counting of derivatives and the pion mass, and predictions to a given order are generated from tree diagrams using vertices from the Lagrangian to that order, and from pion loops involving vertices of up to two orders lower. The divergences from loops are consistently absorbed into the low-energy constants (LECs) at the relevant order. The field theory at its simplest contains only pions and nucleons; the effects of heavier mesons and nucleon excitations, being short-range, are encoded in the LECs.

A major advantage of an EFT is the ability to use the power-counting to estimate theoretical uncertainties. Though recognised from the earliest days, there has been a recent upsurge in interest in this point. A natural framework is the Bayesian one, see Ref. [14].

Of course in such a theory we give up any attempt to model quark substructure of hadrons. Any property which is dominated by such short-distance physics (the anomalous magnetic moments of the nucleons is an example) is encoded in low-energy constants in the Lagrangian. The electric polarisability on the other hand arises primarily from pionic fluctuations, and indeed would diverge in the chiral limit. It turns out to be very well described in leading-order $\mathrm{HB} \chi \mathrm{PT}$, as was famously demonstrated by Bernard et al. a number of years ago now [15]. The magnetic polarisability on the other hand seems to have comparable contributions from both longer and shorter distance physics. However working to NNLO in $\mathrm{HB} \chi \mathrm{PT}$ allows for prediction of the full set of six energy- and scattering-angle-dependent amplitudes of Compton scattering from the nucleon in terms of only 


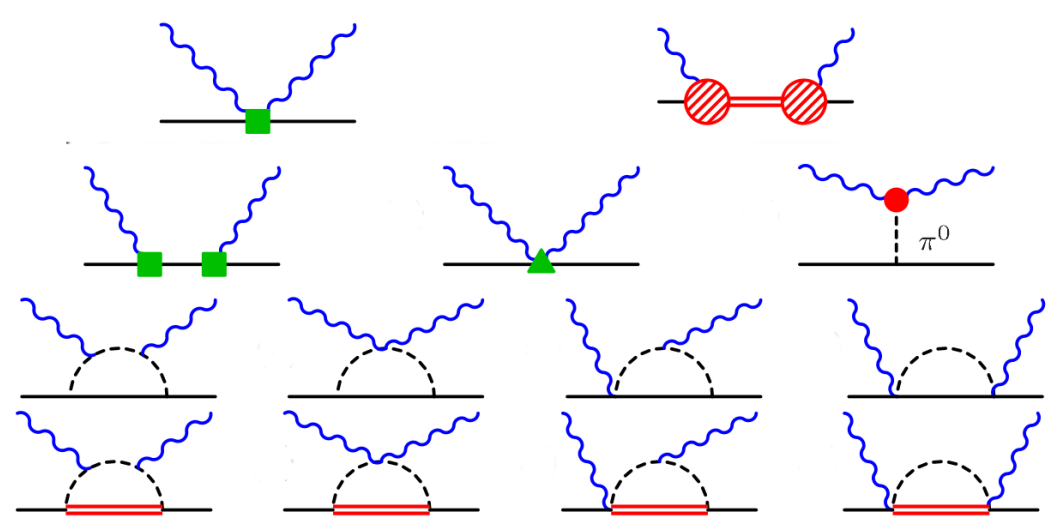

Figure 1: (Colour online) Tree, $\Delta(1232)$ pole, leading $N \pi$, and $\Delta \pi$ loop diagrams that contribute to Compton scattering. The double line represents the $\Delta(1232)$. The fourth-order $\mathrm{N} \pi$ loop diagrams are included but not depicted. Permuted and crossed diagrams not shown. Figure adapted from Ref. [11].

two free parameters, namely the fourth-order LECs which constitute the leading short-distance contribution to the electric and magnetic polarisabilities [16]. A fit of these parameters to data is equivalent to an extraction of the polarisabilities, as we now describe.

\section{Compton scattering from the nucleon}

Chiral descriptions of Compton scattering [13, 17], and extractions of polarisabilities [18, 19], date back some years. Details of the calculations reported here are given in Ref. [11] including the specification of the chiral Lagrangian used. One important point is that we included the $\Delta(1232)$ as an explicit degree of freedom;. Its excitation energy is not much more than $2 m_{\pi}$, and a cursory glance at Compton scattering data shows its overwhelming importance above about $200 \mathrm{MeV}$ photon energy. The power counting that obtains in the low-energy region is no longer correct around the Delta peak [20] and our theory there is only good to NLO; hence we only use data from the resonance region to constrain the Delta parameters and not for our fits.

Ref. [1] contains a critical evaluation of the data for Compton scattering from the proton below about $200 \mathrm{MeV}$, they are quite numerous but not all consistent, and some degree of selection was required. Our results for $\alpha_{E 1}^{(\mathrm{p})}$ and $\beta_{M 1}^{(\mathrm{p})}$ were compatible with the Baldin sum rule $\alpha_{E 1}^{(\mathrm{p})}+\beta_{M 1}^{(\mathrm{p})}=$ $13.8 \pm 0.4$, so we reduced our statistical errors by imposing that constraint, giving

$$
\begin{aligned}
& \alpha_{E 1}^{(\mathrm{p})}=10.65 \pm 0.35(\mathrm{stat}) \pm 0.2(\text { Baldin }) \pm 0.3 \text { (theory) }, \\
& \beta_{M 1}^{(\mathrm{p})}=3.15 \mp 0.35(\text { stat }) \pm 0.2 \text { (Baldin) } \mp 0.3 \text { (theory). }
\end{aligned}
$$

The predictions of the chiral theory with these fit parameters are shown together with world data in Fig. 2. Work on fitting polarisabilities to world data has also been done recently in dispersionrelation [21] and low-energy-expansion [22] frameworks. The proton database contains experiments over 5 decades which use a huge variety of methods and a wide range of levels of documentation, analysis rigour and self-consistency checks. "Pruning" proves necessary. It appears that different prescriptions lead to statistically only marginally significant discrepancies between 

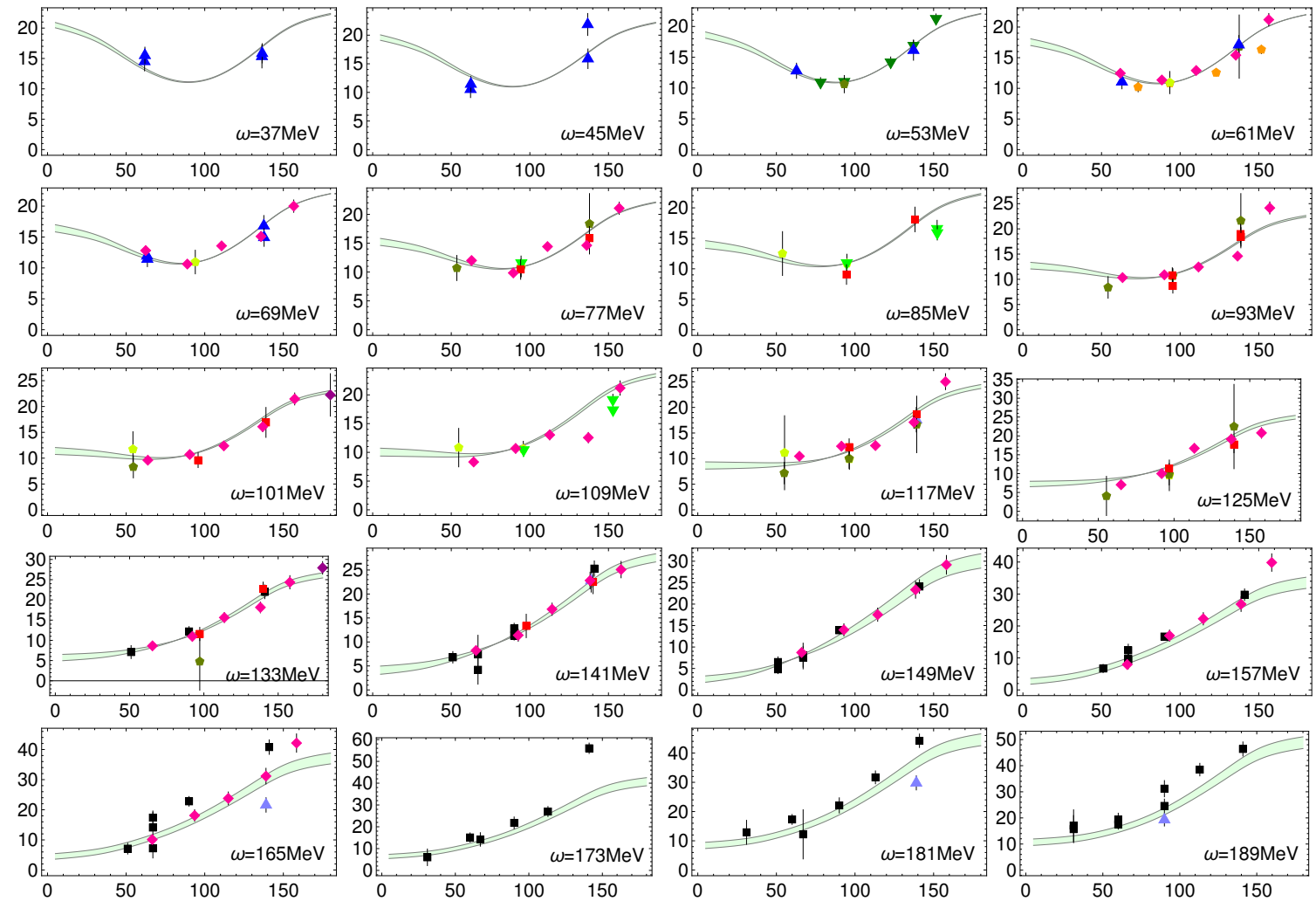

Figure 2: (Colour online) World proton Compton scattering data and chiral EFT predictions. The labelled photon lab energy is the central value of $8 \mathrm{MeV}$ bins; the shaded bands span the same range (variation due to the errors on the extracted polarisabilities is smaller). Symbols are explained in Table 3.1 of Ref. [1].

the higher $\beta_{M 1}^{(\mathrm{p})}$ given above and some extractions which favour a somewhat lower value. There are indications that this may be due not to the theory framework used, but due to the way data are assessed. We refer the reader to the interesting studies by Pasquini et al. [21, 23]. What is needed to resolve this issue is not just more proton data, but data whose statistics and systematics is reproducibly shown to be superior by careful documentation and vetting to show self-consistency, using modern experimental tools and statistical methods.

\section{Compton Scattering from the Deuteron}

From a nuclear-physics point of view, a great merit of the chiral perturbation theory framework is that it allows for a description of nuclear forces from a starting-point which is better aligned with QCD than the traditional approaches, and very substantial progress has been made in describing light and even medium-mass nuclei starting with chiral forces that also do a good job of describing few-body systems (see for example [24]). In the current context the ability to describe the contribution of pions to both nucleon and nuclear Compton scattering on the same footing is of immense benefit. The extension of the work described above to light nuclei, therefore, has two aims: one is to extract the neutron polarisabilities in the absence of a free target, but the other, perhaps more relevant for this conference, is to test the efficacy of a chiral description of nuclear forces to describe 
(a):

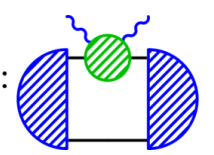

(b):

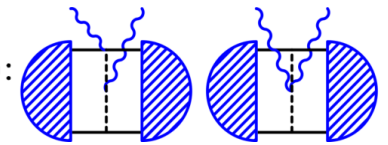

$(c)$

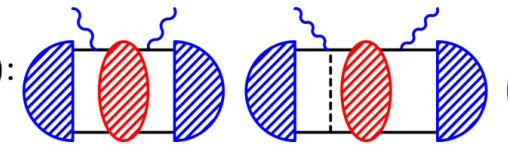

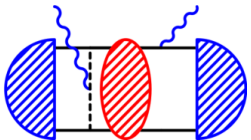

Figure 3: (Colour online) Diagrams that contribute to Compton scattering on the deuteron. The green blob in the one-body diagram (a) represents the graphs of Fig. 1 while the red blob in (c) represents the full NN rescattering, including no interaction. The blue hemisphere represents the deuteron wave function. Figure adapted from Ref. [1]

a dynamical process, by comparing to data and, if a good description can be given, comparing the neutron polarisabilities extracted from different targets.

The various diagrams which contribute are shown in Fig. 3. At chiral energies $E_{\gamma} \sim m_{\pi}$ the rescattering diagram (c) is higher-order. However at very low energies the same enhancement of the two-nucleon propagator that gives rise to the existence of a bound state in the first place requires this graph to be included. Only with the complete set is the correct Thomson limit reproduced; (a) and (b) alone give a result which is out by around a factor of two. As required by the power-counting the contribution of (c) rapidly diminishes with energy. However given that the $\mathrm{NN}$ force used in practice has regulators which introduce momentum-dependence beyond that arising from the Lagrangian, the inclusion of (c) even at higher energies allows consistency with Siegert's theorem and markedly reduces the spread between the results using different forces [25].

In Ref. [1] we fitted $\alpha_{E 1}^{(\mathrm{s})}$ and $\beta_{M 1}^{(\mathrm{s})}$ to the pre-2014 world deuteron data, then in Ref. [6] the fit was updated to include the new MAX-Lab data which almost doubled the size of the database. The one-body diagrams were implemented to NNLO, rather than $\mathrm{N}^{3} \mathrm{LO}$ as in the proton case, and the main consequence of this lower-order fit is a larger theory error than for the proton; however the statistical error still dominates. The isoscalar Baldin sum rule of $\alpha_{E 1}^{(\mathrm{s})}+\beta_{M 1}^{(\mathrm{s})}=14.5 \pm 0.4$, [26] was used as a constraint. We obtained $\alpha_{E 1}^{(\mathrm{s})}-\beta_{M 1}^{(\mathrm{s})}=7.8 \pm 1.2$ (stat) \pm 0.8 (th), with a $\chi^{2}$ of 45.2 for 44 degrees of freedom.

This was then combined with the proton value to extract numbers for the neutron:

$$
\begin{aligned}
& \alpha_{E 1}^{(\mathrm{n})}=11.65 \pm 1.25(\mathrm{stat}) \pm 0.2(\text { Baldin }) \pm 0.8 \text { (theory), } \\
& \beta_{M 1}^{(\mathrm{n})}=3.55 \mp 1.25(\text { stat }) \pm 0.2(\text { Baldin }) \mp 0.8 \text { (theory), }
\end{aligned}
$$

The world data and chiral EFT cross sections are shown in Fig. 4.

As can be seen from Fig. 4, the description of the data is good. Even more so than with the proton, the inclusion of the Delta is required to reproduce the backwards angle cross section, which otherwise falls too low. The results for the polarisabilities are very close to those of the proton (as expected in chiral perturbation theory, since the dominant effects are isoscalar) and indeed a significant isovector contribution is driven entirely by the Baldin sum rule and would be absent in a two-parameter fit.

\section{Compton Scattering from ${ }^{3} \mathrm{He}$}

In principle the calculation for ${ }^{3} \mathrm{He}$ mirrors that of the deuteron, and the first studies were done by Shukla (née Choudhury) Nogga and Phillips [27, 28] and improved and extended by Margaryan 


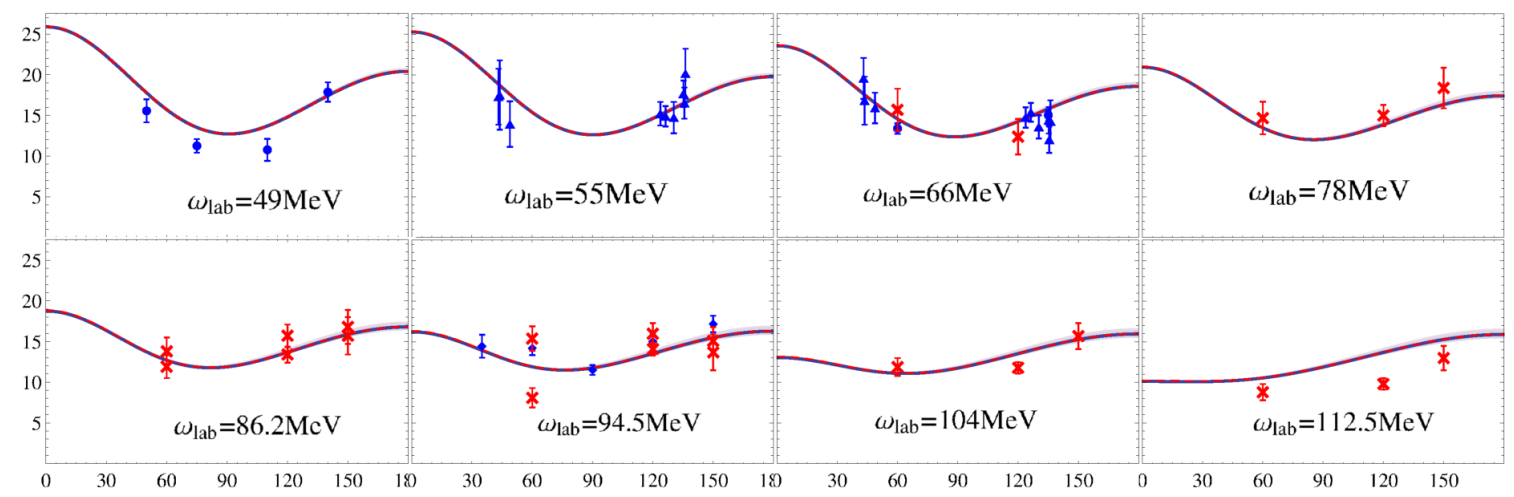

Figure 4: World deuteron Compton scattering data and the chiral EFT fit. The new data of Ref. [6] are the red crosses. Figure adapted from Ref. [6]

et al. [29]. This is the subject of another contribution in these proceedings [12], so we will not show any results here. We will just comment that the equivalent of diagram (c) in Fig. 3, in which the three nucleons rescatter between absorbing and emitting the photons, is not yet included. Based on experience with the deuteron we expect that for energies above $50-60 \mathrm{MeV}$ the results will still be qualitatively reliable, but work is in progress to correct this deficiency.

\section{Spin Polarisabilities}

The effective Hamiltonian of (1.1) contains not only the easily-interpreted electric and magnetic polarisabilities, but also four spin-dependent polarisabilities. Because their contributions to the low-energy amplitudes go as $\omega^{3}$, and also because they are not enhanced by interference with the leading Thomson amplitude, their influence on the cross section is rather small. They are predicted to the order at which we work in $\chi \mathrm{EFT}$, and the existing data does not provide enough sensitivity to extract them without further constraints (see also [22]). A summary of our knowledge of these is given in Refs $[10,30]$ and repeated here for our $\chi$ EFT variant.

\begin{tabular}{|r||l|l|}
\hline & proton & neutron \\
\hline \hline$\gamma_{E 1 E 1}$ & $-1.1 \pm 1.9_{\text {theory }}$ & $-4.0 \pm 1.9_{\text {theory }}$ \\
$\gamma_{M 1 M 1}$ & $2.2 \pm 0.5_{\text {stat }} \pm 0.6_{\text {theory }}$ & $1.3 \pm 0.5_{\text {stat }} \pm 0.6_{\text {theory }}$ \\
$\gamma_{E 1 M 2}$ & $-0.4 \pm 0.6_{\text {theory }}$ & $-0.1 \pm 0.6_{\text {theory }}$ \\
$\gamma_{M 1 E 2}$ & $1.9 \pm 0.5_{\text {theory }}$ & $2.4 \pm 0.5_{\text {theory }}$ \\
\hline
\end{tabular}

Table 1: The dipole spin polarisabilities of the proton and neutron in $\chi$ EFT with explicit $\Delta(1232)$ degrees of freedom at $\mathscr{O}\left(e^{2} \delta^{4}\right)$, in $10^{-4} \mathrm{fm}^{4}[11,10]$.

Here, the proton spin polarisability $\gamma_{M 1 M 1}^{(\mathrm{p})}$ has been fitted as described in ref. [11], but $\gamma_{M 1 M 1}^{(\mathrm{n})}$ is then predicted from that. A thorough discussion of all aspects, including estimates of residual theoretical uncertainties in a Bayesian framework, can be found in ref. [10].

Given the scarcity of data, finding observables which are sensitive to particular combinations of spin polarisabilities is for now more important than quoting central values. Fortunately, sensi- 
tivity studies are quite insensitive to the particular central values of spin and scalar polarisabilities chosen - pun intended.

The natural observables for this purpose are those obtained with polarised targets and photons. Few such experiments have been done, and only for the proton. Some are at energies sufficiently high (around $280 \mathrm{MeV}$ lab energy) that realistic theory errors on polarisability extractions in a chiral framework would be very large. As an indication of this, it is helpful to consider the so-called "dynamical polarisabilities", which are linear combinations of Compton scattering multipoles, with the leading dependence on $\omega$ factored out, so that the $\omega \rightarrow 0$ limit coincides with the corresponding (static) polarisability (see $[19,30]$ and references therein). Fig. 5 shows the the first 8 multipoles for Proton Compton scattering in the present theory (after fitting as described above), in the $\mathscr{O}\left(e^{2} \delta^{3}\right)$ covariant framework of Lensky et al. [30] (without fitting to data), and in the dispersion-relation framework of Pasquini et al. [19], based on integrals over pion-photoproduction multipoles (with $\alpha_{E 1}-\beta_{M 1}$ and $\gamma_{\pi}$ fit to Compton scattering data). Varying a static polarisability is identical to simply shifting the corresponding dynamical polarisability up or down.

For low-energy scattering, the message of this plot is very positive. There is a substantial degree of agreement on the shape of the polarisabilities among the approaches up to around $250 \mathrm{MeV}$ lab energy ( $200 \mathrm{MeV} \mathrm{cm}$ energy in the figure). Furthermore, after adjusting the static polarisabilities to a common value, the results generically lie very close to one another. Indeed, the same pion-loop and Delta-resonance physics is encoded in all three calculations. In the Delta-dominated multipoles, this agreement continues up to surprisingly high energies, but overall disagreement becomes quite pronounced above $250 \mathrm{MeV}$ lab energy. Thus at these energies, a reliable link between the amplitudes and the static polarisability is lost.
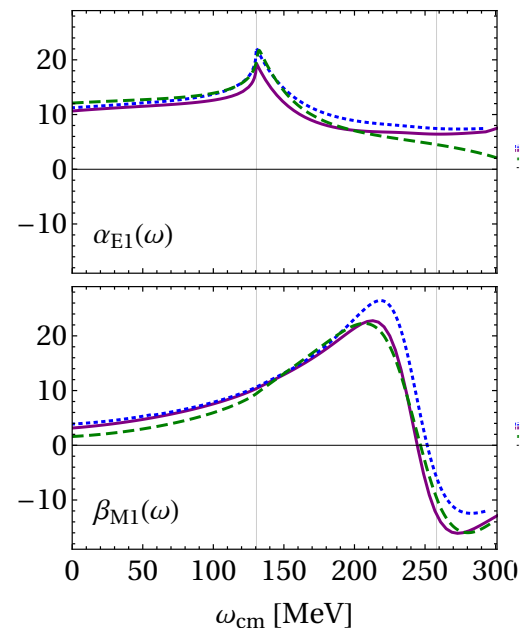
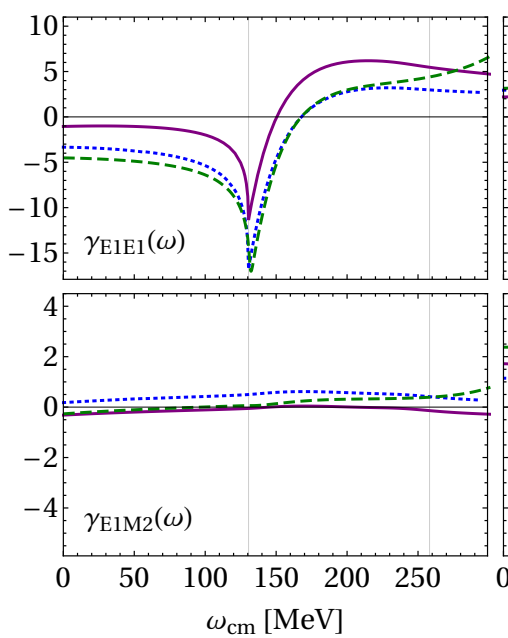

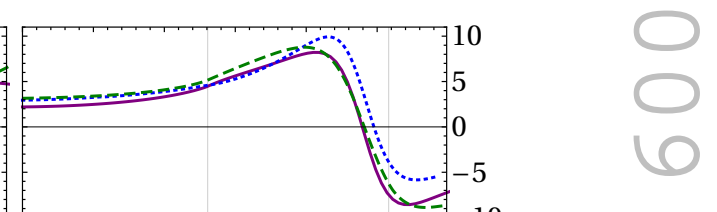

Figure 5: (Colour online) Real parts of the dominant dynamical polarisabilities for low-energy Compton scattering from the proton, plotted as a function of $\mathrm{cm}$ photon energy. The units are $10^{-4} \mathrm{fm}^{n}$ where $n=3$ for $\alpha_{E 1}$ and $\beta_{M 1}, n=4$ for the $\gamma_{i}$, and $n=5$ for $\alpha_{E 2}$ and $\beta_{E 2}$. Red (solid): this work; green (dashed): DRbased by Pasquini et al. [31]; blue (dotted) 3rd-order covariant $\chi$ PT by Lensky et al. [30]. Note that each row has its distinct plot scale. Figure adapted from [9].

In our recent paper [9] we explored the sensitivity of various target-beam asymmetries to the spin polarisabilities. As an advertisement for our results we here show the prediction for the asym- 
metry $\Sigma_{2 x}$, which involves circularly polarised photons and a target polarised perpendicularly to the scattering plane. A grey mist over the high-energy, right-hand end of the plots indicates the region where $\chi$ EFT cannot be expected to converge, which is also the region where different theoretical approaches start to diverge; see above. Unfortunately, that is the region where much of the data was taken, but it is remarkable how well theory and data agree. In Fig. 6, we compare the observable itself with data from MAMI [4]. In Fig. 7, we show "heat-plots" of the sensitivity to several polarisabilities and their combinations. The colour indicates the derivative of the observable with respect to the polarisability (or combination), with deep red and deep blue indicating large positive and negative values respectively. This observable is particularly sensitive to the combination $\gamma_{E 1 E 1}-\gamma_{E 1 M 2}$, with little confounding sensitivity to other combinations if the Baldin Sum rule is used to fix $\alpha_{E 1}+\beta_{M 1}$. The plot also shows that the extant data (circles) is not at the best kinematics (even leaving aside the theory disagreement at such high energy). For a fuller explanation of the plots, the reader is referred to the original paper.
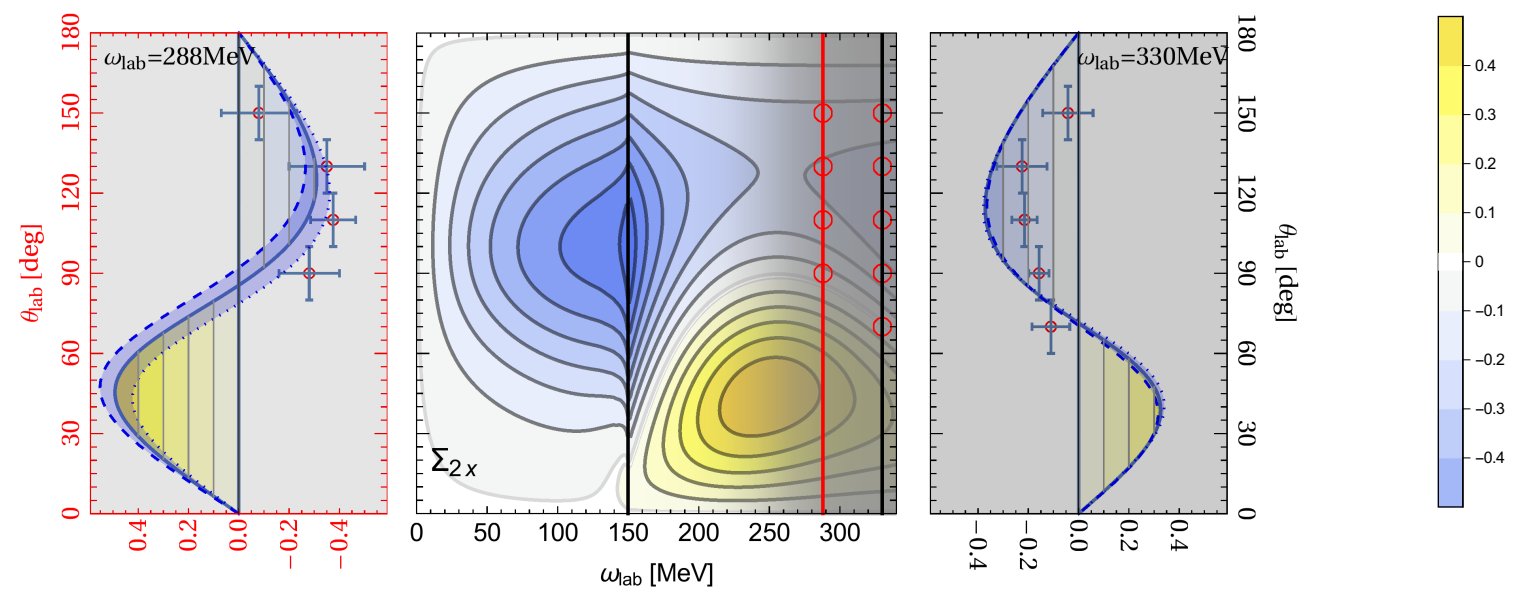

Figure 6: (Colour online) The double asymmetry $\Sigma_{2 x}$ (circularly polarised photons on a proton target polarised along the $x$ axis); see text for details. Circles indicate the approximate location of the data of [4], which are compared to at the wings. Figure modified from [9].

\section{Summary}

In conclusion, Chiral Effective Field Theory provides a highly effective tool for the exploration of Comprton scattering on light nuclei. Up-coming analyses and new experiments at MAMI and $\mathrm{HI} \gamma \mathrm{S}$ will provide accurate date on several targets, so that multiple extractions of neutron polarisabilities should soon be possible, along with further confirmation of the extent to which the same theory describes few-body system in a systtematically improvable framework.

\section{Acknowledgements}

This work was supported in part by the US Department of Energy under contracts DE-SC0015393 (HWG) and DE-FG02-93ER-40756 (DRP), and by UK Science and Technology Facilities Council grants ST/L005794/1 and ST/P004423/1 (JMcG). 


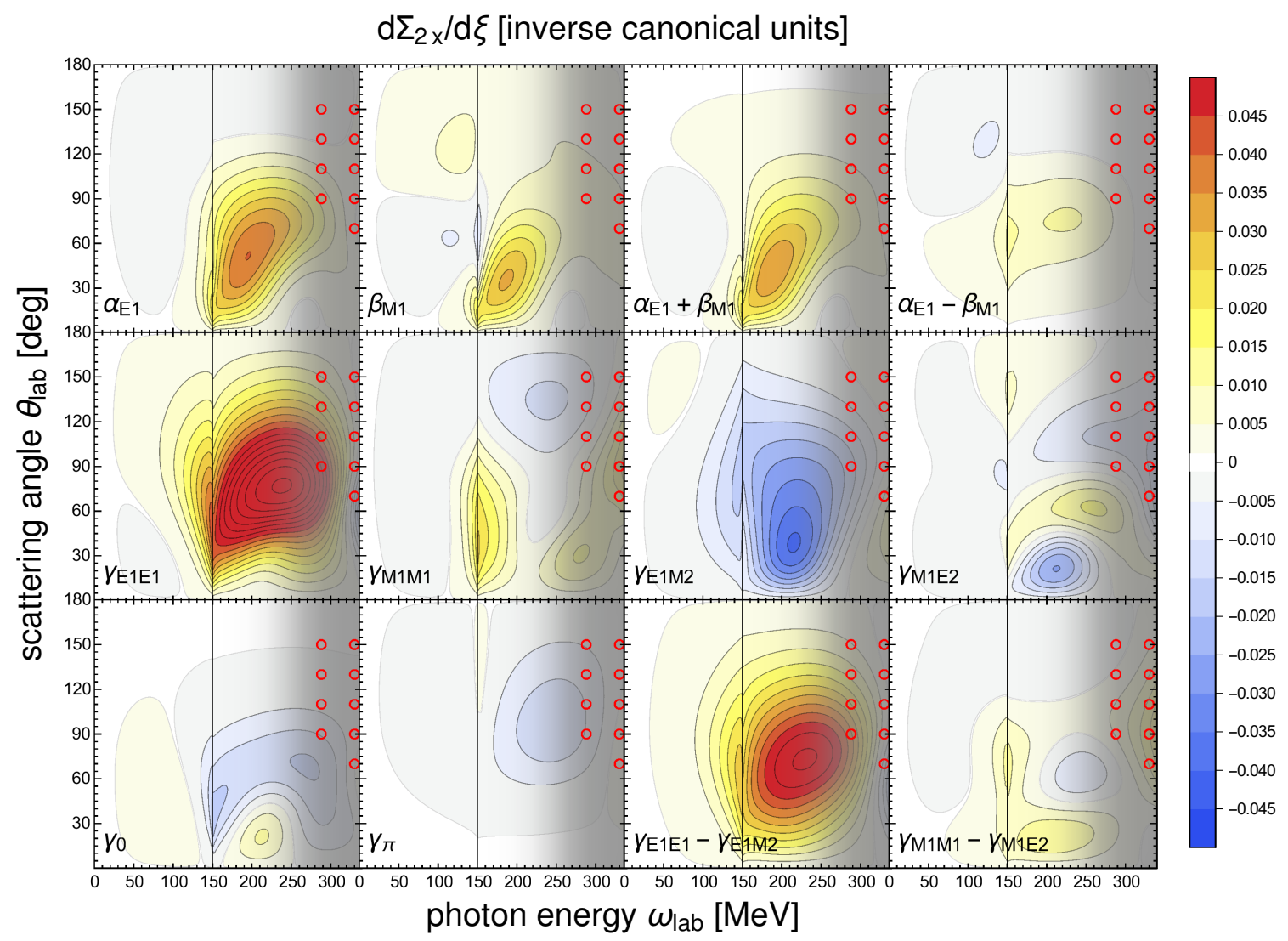

Figure 7: (Colour online) Sensitivity of the double asymmetry $\Sigma_{2 x}$ (circularly polarised photons on a proton target polarised along the $x$ axis) to varying the polarisabilities; see text for details. Circles indicate the approximate location of the data of [4]; their size does not reflect the error bars, nor the size of energy or angle bins. Figure modified from [9].

\section{References}

[1] Grießhammer H. W., McGovern J. A., Phillips D. R. and Feldman G., Prog. Part. Nucl. Phys. 67 (2012) 841. [arXiv:1203.6834 [nucl-th]].

[2] Babusci D., Giordano G., L'vov A. I., Matone G. and Nathan A. M., Phys. Rev. C 58 (1998) 1013. [arXiv:hep-ph/9803347].

[3] See the contributions to these proceedings by D. Hornidge, E. Downie, A. Alexandru, Ph. Martel, M. Sikora, L. Myers, R. Miskimen, C. Howell, M. Hoferichter, A. Walker-Loud, R. Young and V. Lensky.

[4] Martel P. P. et al. [A2 Collaboration], Phys. Rev. Lett. 114, no. 11, 112501 (2015) [arXiv:1408.1576 [nucl-ex]].

[5] Huber G. M. and Collicott C., arXiv:1508.07919 [nucl-ex].

[6] Myers L. S. et al. [COMPTON@MAX-lab Collaboration], Phys. Rev. Lett. 113, no. 26, 262506 (2014) [arXiv:1409.3705 [nucl-ex]].

[7] Sokhoyan V. et al. [A2 Collaboration], Eur. Phys. J. A 53, no. 1 (2017) 14 [arXiv:1611.03769 [nucl-ex]]. 
[8] Sikora M. H. et al., Phys. Rev. C 96 (2017) 055209.

[9] Grießhammer H. W., McGovern J. A. and Phillips D. R., Eur. Phys. J. A 54 (2018) no.3, 37 [arXiv:1711.11546 [nucl-th]].

[10] Grießhammer H. W., McGovern J. A. and Phillips D. R., Eur. Phys. J. A 52, no. 5 (2016) 139 [arXiv:1511.01952 [nucl-th]].

[11] McGovern J. A., Phillips D. R. and Grießhammer H. W., Eur. Phys. J. A 49 (2013) 12 [arXiv:1210.4104 [nucl-th]].

[12] Grießhammer H., McGovern J. A. and Phillips D. R., these proceedings.

[13] Bernard V., Kaiser N. and Meißner U. G., Int. J. Mod. Phys. E 4 (1995) 193 [arXiv:hep-ph/9501384].

[14] Wesolowski S., Klco N., Furnstahl R. J., Phillips D. R. and Thapaliya A., J. Phys. G 43 (2016) no.7, 074001 doi:10.1088/0954-3899/43/7/074001 [arXiv:1511.03618 [nucl-th]].

[15] Bernard V., Kaiser N., Kambor J. and Meißner U. G., Nucl. Phys. B 388 (1992) 315.

[16] Bernard V., Kaiser N., Schmidt A. and Meißner U. G., Phys. Lett. B 319 (1993) 269 [arXiv:hep-ph/9309211].

[17] McGovern J. A., Phys. Rev. C 63 (2001) 064608 [Erratum-ibid. C 66 (2002) 039902] [nucl-th/0101057].

[18] Beane S. R., Malheiro M., McGovern J. A., Phillips D. R. and van U. Kolck, Nucl. Phys. A 747 (2005) 311 [arXiv:nucl-th/0403088].

[19] Hildebrandt R. P., Grießhammer H. W., Hemmert T. R. and Pasquini B., Eur. Phys. J. A 20 (2004) 293 [arXiv:nucl-th/0307070].

[20] Pascalutsa V., Phillips D. R., Phys. Rev. C67 (2003) 055202. [nucl-th/0212024].

[21] Pasquini B., Pedroni P. and Sconfietti S., [arXiv:1711.07401 [hep-ph]].

[22] Krupina N., Lensky V. and Pascalutsa V., [arXiv:1712.05349 [nucl-th]].

[23] Pasquini B., Pedroni P. and Sconfietti S., [arXiv:1903.07952 [hep-ph]].

[24] Lonardoni D., Gandolfi S., Lynn J. E., Petrie C., Carlson J., Schmidt K. E. and Schwenk A., Phys. Rev. C 97 (2018) no.4, 044318 [arXiv:1802.08932 [nucl-th]].

[25] Hildebrandt R. P., Grießhammer H. W. and Hemmert T. R., Eur. Phys. J. A 46 (2010) 111 [arXiv:nucl-th/0512063].

[26] Levchuk M. I. and L’vov A. I., Nucl. Phys. A 674 (2000) 449. [arXiv:nucl-th/9909066].

[27] Choudhury D., Nogga A. and Phillips D. R., Phys. Rev. Lett. 98 (2007) 232303 [arXiv:nucl-th/0701078].

[28] Shukla D., Nogga A. and Phillips D. R., Nucl. Phys. A 819 (2009) 98 [arXiv:0812.0138 [nucl-th]].

[29] Margaryan A., Strandberg B., Grießhammer H. W., Mcgovern J. A., Phillips D. R. and Shukla D., Eur. Phys. J. A 54 (2018) no.7, 125 [arXiv:1804.00956 [nucl-th]].

[30] Lensky V., McGovern J. A. and Pascalutsa V., Eur. Phys. J. C 75 (2015) no.12, 604 [arXiv:1510.02794 [hep-ph]].

[31] Pasquini B., private communication based on Ref. [19]. 